\title{
DETERMINING CABLES METRICS USING 3D ULTRASONIC SCANNING
}

\author{
Simon SUTTON \\ University of Southampton, UK \\ s.sutton@southampton.ac.uk
}

\author{
Paul WILLMOTT \\ Acuity Products Ltd, UK \\ paul.willmott@acuityproducts.co.uk
}

\begin{abstract}
An examination of XLPE cable samples is reported using an ultrasonic scanning technique which potentially offers improved quality assurance for cable buyers. Dimensional parameters are reported along with a more detailed investigation of variations at the inner semiconinsulation interface which correlate with characteristics of the cable construction.
\end{abstract}

\section{INTRODUCTION}

The reliability of a cable relies on a combination of many factors; quality of materials, manufacturing quality and control, and care during jointing and installation. Since the introduction of extruded cables in the 1960s advances in all of these areas has vastly improved the service reliability of distribution and transmission cables. Nevertheless, customers' expectations for reliable electricity continues to rise, as do the potential penalties imposed by regulators for missing service targets. Additionally, cables are now also being used in situations with even higher reliability expectations; for example, HVDC interconnections and offshore windfarm cables (array cables and connections to shore). In both these cases cable failures require long repair times and result in significant lost revenue.

Manufacturing quality and control even at the highest operating voltages currently relies on short cable samples (typically less than $30 \mathrm{~cm}$ ) taken from the end of every drum length which undergo dimensional checks (for example the thicknesses of the extruded layers) and a hotoil bath inspection to detect contaminants and screen imperfections. Additionally, routine factory testing with partial discharge detection is applied to the whole cable length to detect gross defects, however experience shows this may fail to find well-bonded defects. This regime has served the industry well but means only a very small percentage of the cable is ever measured or visually examined: the statistical significance of these measurements gets worse as drum lengths increase and risks missing infrequent defects or deviations from the cable specification. For example, for a one kilometre drum length less than $0.05 \%$ of the cable is examined/measured, and for a large subsea cable this falls to just $0.001 \%$.

In this study we report on findings from a new ultrasonic scanning technique capable of either continuously inspecting and measuring the cable core during manufacture or detailed offline analysis.

\section{MEASUREMENT SYSTEM}

Data have been collected using an UltraProfilor (Acuity Products Ltd) which is an offline instrument for performing investigations of short sections of cable. For these measurements the cable is static, suspended in a tank of water and the scanning head is moved along the cable at a chosen speed: this determines the longitudinal resolution of the measurements. A similar instrument can be installed on a cable production line (UltraScreen), in which case the scanning head is fixed and the cable core moves through the scanning head (Figure 1) at the line-speed of cable production. More details of the measurement procedure and data processing are given in [1].

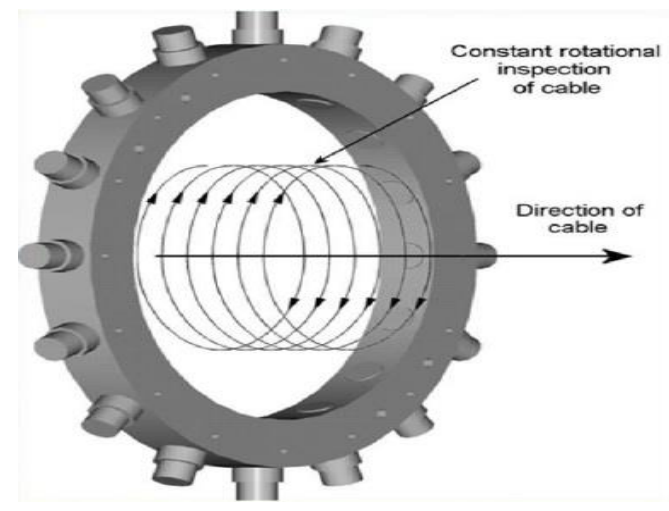

Figure 1: Diagram of UltraScreen scanning head surrounding a cable core.

Ultrasonic measurement techniques detect the interface between materials of different acoustic impedence. Each transducer around the scanning head provides the radial position of the four interfaces; water/outer semicon, outer semicon/insulation, insulation/inner semicon and inner semicon/conductor. These data are then used to calculate the thicknesses of the three cable component layers to an accuracy of $\sim 10-20 \mu \mathrm{m}$ : data from opposing transducers are used to calculate the diameter of the cable. Many cable quality parameters can be extracted from the data including concentricity and eccentricity.

\section{DIMENSIONAL MEASUREMENTS}

Three cables with conductor sizes of 185, 630 and $2500 \mathrm{~mm}^{2}$, and rated at 33,33 and $400 \mathrm{kV}$ respectively (Figure 2) have been scanned using an UltraProfilor. Approximately one metre of each cable type has been examined during this study. 


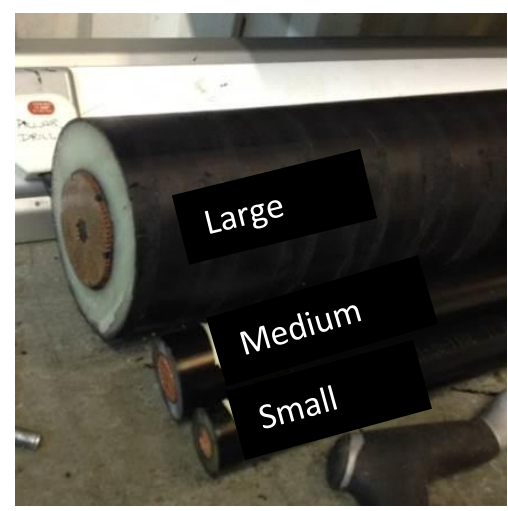

Figure 2: Photo of the three cables examined, 185, 630 and $2500 \mathrm{~mm}^{2}$.

The cables were scanned at $40 \mathrm{~cm} / \mathrm{min}$, which corresponds to approximately one measurement every $100 \mu \mathrm{m}$ along the length of the cable sample. The instantaneous thickness of the two semicon layers and insulation was measured for all three cables, and the concentricity was also calculated (Figure 3). Table 1 shows the data for all three cables at particular locations.

\begin{tabular}{|l|c|c|c|c|}
\hline \multirow{2}{*}{$\begin{array}{l}\text { Cable } \\
\left(\mathbf{m m}^{2}\right)\end{array}$} & \multicolumn{2}{|c|}{ Average thickness (mm) } & Concentricity \\
\cline { 2 - 4 } & $\begin{array}{c}\text { Inner } \\
\text { Semicon }\end{array}$ & $\begin{array}{c}\text { Outer } \\
\text { Semicon }\end{array}$ & XLPE & \\
\hline $\mathbf{1 8 5}$ & 0.87 & 0.68 & 7.72 & 6.39 \\
\hline $\mathbf{6 3 0}$ & 1.08 & 0.68 & 7.58 & 5.94 \\
\hline $\mathbf{2 5 0 0}$ & 1.79 & 1.79 & 23.83 & 2.54 \\
\hline
\end{tabular}

Table 1: Typical dimensional parameters for the three cables at single locations.

The average values are calculated from the eight individual measurements taken around the diameter of the cable (left hand side of Figure 3); from these data the concentricity of the cable can be derived. The $400 \mathrm{kV}$ cable is notably more concentric than the two $33 \mathrm{kV}$ cables. Also as would be expected the insulation thickness of the two $33 \mathrm{kV}$ cables is approximately the same.

The graphs to the right hand side of Figure 3 show data along the length of the cable sample. The average values of the layer thicknesses are shown by the green lines, and the instantaneous maximum and minimum thicknesses from all eight measurement points are shown by the blue lines. The minimum thickness is important because this is usually defined in the cable specification. When installed on a manufacturing line besides vastly improving the frequency of dimensional measurements, the continuous scanning is capable of detecting rare anomalies such as semicon protrusions (Figure 4), breaks in the semicon layer or fall-in, or rare anomalies such as contaminants (Figure 5). Such anomalies are normally only identified if, by chance, they occur in the short sample taken for hot-oil inspection.

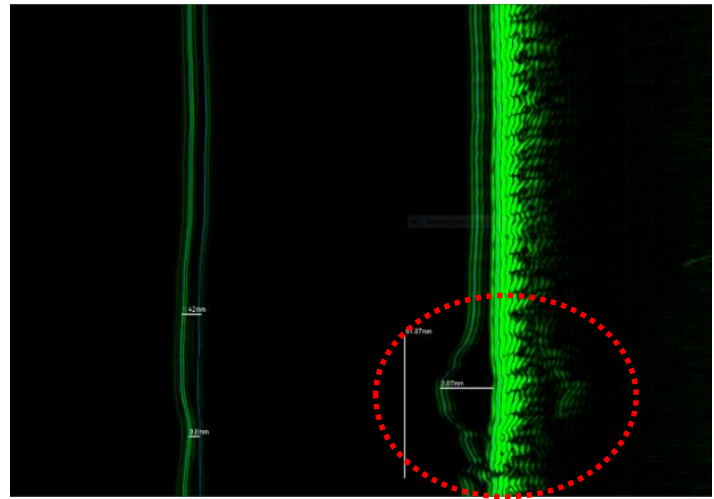

Figure 4: Large protrusion on the inner semicon (not from one of the cables examined in this study).

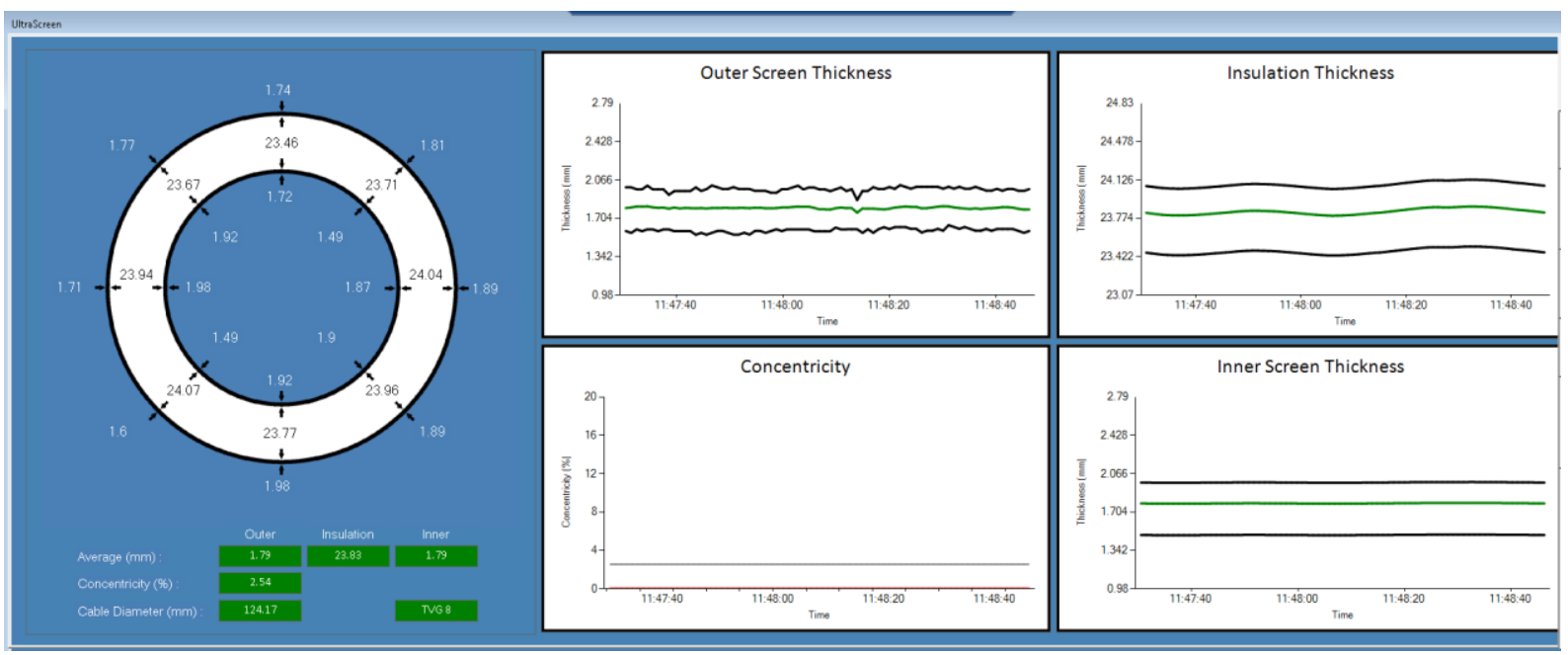

Figure 3: Screen shot of the instantaneous and average data along the cable sample length from the $2500 \mathrm{~mm}^{2} 400 \mathrm{kV}$ cable examined. 


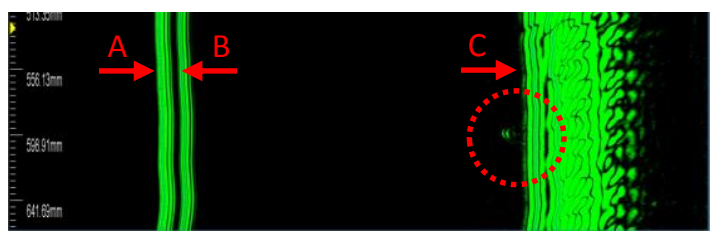

Figure 5: Contaminant identified in the insulation (not from one of the cables examined in this study).

In Figures 4 and 5 the outer surface of the cable is labelled $\mathrm{A}$, this is the water/semicon interface during the measurement; the outer semicon/insulation interface is labelled $\mathrm{B}$ and the inner semicon/insulation interface labelled C. Distance along the cable is increasing from the top of the plot, and each plot only represents the data from one transducer channel. In Figure 4 it can also be observed that after the protrusion on the inner semicon, there is a notable thinning of the outer semicon.

\section{INNER SEMICON/INSULATION INTERFACE}

Previous work has shown that by monitoring the position of the interfaces between the components along the length of the cable, both short and long periodicity fluctuations can be observed. For example, Figure 6 (reproduced from [1]) shows the deviation of the inner semicon/insulation interface

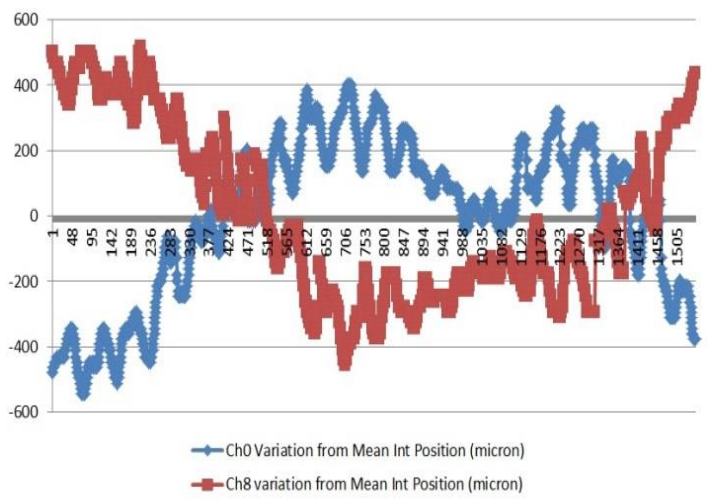

Figure 6: Relative position of the semicon/insulation interface on opposing transducer channels.

In this case $\mathrm{Ch} 0$ is the transducer at the top of the cable and Ch8 is diametrically opposite at the bottom. The low frequency variation shows that the cable conductor is moving up and down within the cable (thereby causing concentricity variations) over a length of $\sim 0.8 \mathrm{~m}$ : the conductor is "high" relative to the central position on the left hand side of Figure 6 before sinking below the central position and then rises again on the right hand side. Additionally there are also higher frequency oscillations showing variations on much more local scale.

All three cables have been scanned and the smoothness of the inner semicon/insulation interface extracted from the data. Figure 7 shows a detailed scan from a $20 \mathrm{~cm}$ length of the $630 \mathrm{~mm}^{2} 33 \mathrm{kV}$ cable. Measurements were taken approximately every $100 \mu \mathrm{m}$ along the cable. A clear periodicity is evident in the data. Similar variations are seen on all the other transducer channels and in the other two cables. Consequently this variation is not cable or manufacturer specific.

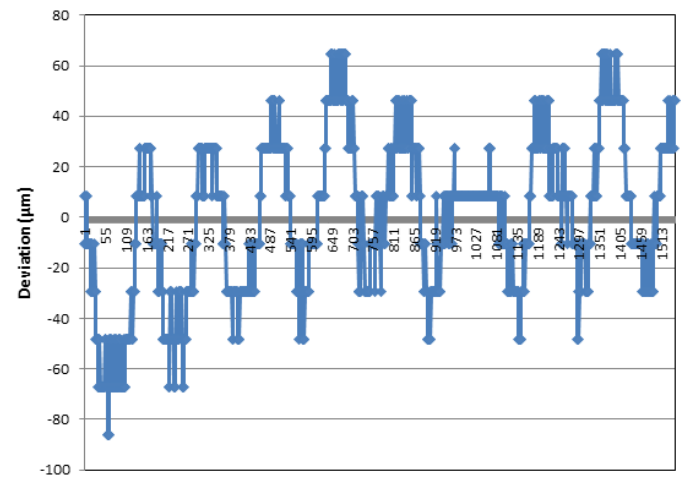

Figure 7: Relative variation in the inner semicon surface of the $630 \mathrm{~mm}^{2} 33 \mathrm{kV}$ cable.

Figure 7 is plotted as a succession of measurements, i.e. the $\mathrm{x}$-axis has not been converted to distance. Moreover, the data are noisy since the total deviations are small, $\pm \sim 60 \mu \mathrm{m}$, and close to the resolution of the instrument $(\sim 20 \mu \mathrm{m})$. It is nevertheless surprising to discover such a regular feature which poses the question what could be the underlying cause. Contrastingly, movement of the conductor within the cable core, such as in Figure 6, might be explained by subtle variations in the tension on the production line, or even natural oscillations set up in the cable core as it moves through the vulcanisation tube.

The data in Figure 7 were analysed using a Fast Fourier Transform to extract the key frequency components (Figure 8). It is possible to convert frequency to length along the cable using the following formula:

\section{Length $=$ Scan Speed $/$ Frequency}

Thus for the observed frequency component at $0.36 \mathrm{~Hz}$ when the scanning speed was $0.4 \mathrm{~m} / \mathrm{min}$, this produces a corresponding periodicity in the inner semicon surface along the cable length of $1.85 \mathrm{~cm}$.

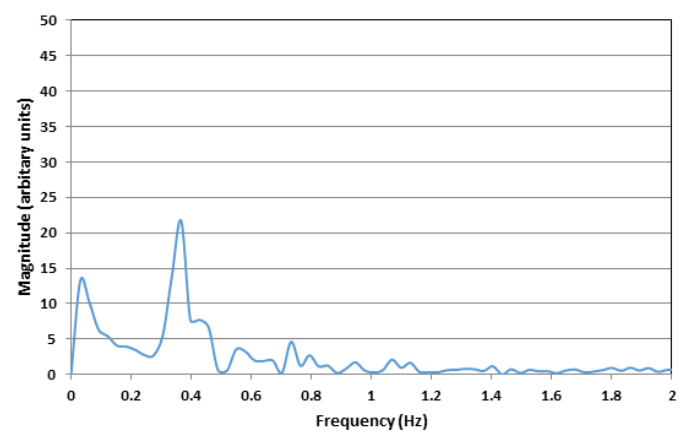

Figure 8: Fast Fourier Transform of the data in Figure 7. 
Table 2 shows the corresponding analysis of the other two cables. The wavelength of the longitudinal variation differs between the cables and does not correlate with either the conductor size or voltage class.

\begin{tabular}{|c|c|c|}
\hline $\begin{array}{c}\text { Cable } \\
\left(\mathbf{m m}^{2}\right)\end{array}$ & $\begin{array}{c}\text { Frequency } \\
(\mathbf{H z})\end{array}$ & $\begin{array}{c}\text { Wavelength } \\
(\mathbf{c m})\end{array}$ \\
\hline $\mathbf{1 8 5}$ & 0.49 & 1.36 \\
\hline $\mathbf{6 3 0}$ & 0.36 & 1.85 \\
\hline $\mathbf{2 5 0 0}$ & 0.40 & 1.68 \\
\hline
\end{tabular}

Table 2: Wavelength of the longitudinal variation on the inner semicon surface.

All three cables have conductors comprising compacted circular copper wires although the strand size varies between cables: the $400 \mathrm{kV}$ cable has a Milliken design. Stripping the cable back to the bare conductor reveals that the observed longitudinal periodicity is related to the helical pitch of the conductor strands (Figure 9). The distance, $\lambda$, corresponds to the longitudinal spacing between conductor strands along the cable.

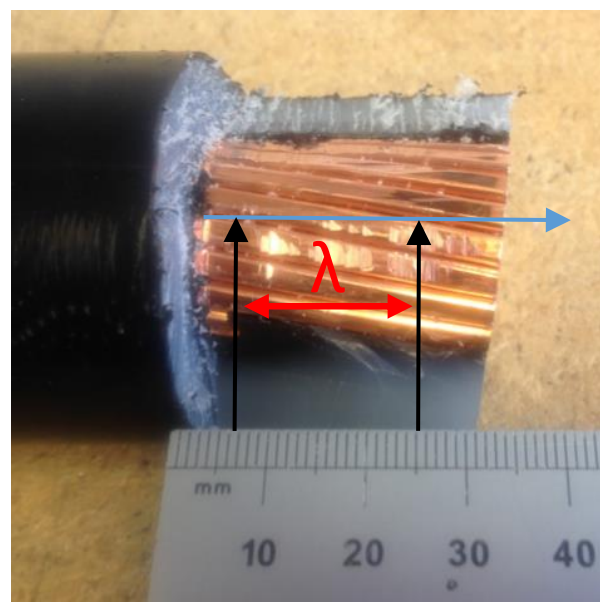

Figure 9: Photo showing the longitudinal distance between successive conductor strands $(\lambda)$ of the $630 \mathrm{~mm}^{2} 33 \mathrm{kV}$ cable.

Table 3 shows a comparison of the longitudinal spacing between conductor strands $(\lambda)$ in the three cables, simply estimated using a ruler, and the wavelength of the variations in the inner semicon surface: the correlation between the two is remarkably close. It may not be completely surprising that some trace of the stranded nature of the conductor is detectable on the surface of inner semicon of the medium voltage cables, given that the semicon layer is only $\sim 1 \mathrm{~mm}$ thick (Table 1). However for the $400 \mathrm{kV}$ cable, the inner semicon is considerably thicker and yet still displays a longitudinal variation of approximately the same surface roughness $\pm \sim 50 \mu \mathrm{m}$, compared to $\pm \sim 60 \mu \mathrm{m}$ for the $33 \mathrm{kV}$ cables.

\begin{tabular}{|c|c|c|}
\hline $\begin{array}{c}\text { Cable } \\
\left(\mathbf{m m}^{\mathbf{2}}\right)\end{array}$ & $\begin{array}{c}\text { Wavelength } \\
(\mathbf{c m})\end{array}$ & $\begin{array}{c}\boldsymbol{\lambda} \\
(\mathbf{c m})\end{array}$ \\
\hline $\mathbf{1 8 5}$ & 1.36 & 1.5 \\
\hline $\mathbf{6 3 0}$ & 1.85 & 1.8 \\
\hline $\mathbf{2 5 0 0}$ & 1.68 & 1.6 \\
\hline
\end{tabular}

Table 3: Comparison of the measured longitudinal distance between successive conductor strands $(\lambda)$ and wavelength of the variation on the inner semicon surface.

\section{DISCUSSION}

The ability to determine the thicknesses of the layers comprising an extruded cable and other measures of production quality, with far greater frequency along the length of a cable than current quality control measures allow is a significant improvement. Moreover the ability to detect defects in a cable which may successfully pass through routine factory electrical testing, yet fail in service some time later, is a major advance for cable buyers; particularly for critical cables to be installed in inaccessible locations, e.g. offshore.

The discovery of small periodic variations in the surface of the inner semicon surface has revealed that there are still aspects of extruded cables to be uncovered. Examination of further cable specimens may yet identify other variability which can be tied back to the construction of the cable, materials used or the manner in which the cable is produced.

\section{CONCLUSIONS}

Three cables of widely differing conductor size and voltage class have been examined using a new ultrasonic scanning technique. The methodology not only allows continuous measurement of the thicknesses of the component layers but also calculation of parameters such as concentricity and eccentricity. Moreover the method can identify defects such as screen protrusions, fall-in or inclusions in the insulation.

Detailed analysis of the inner screen surface has identified a periodic longitudinal variation in the semicon/insulation interface. The periodicity has been matched to the longitudinal distance between successive conductor strands.

\section{REFERENCES}

[1] G. Humphreys-Jones, 2015, "Ultrasonic extrusion quality monitoring of multilayer $\mathrm{HV}$ cables during production - A New Vision in Cable Monitoring", Proceedings $9^{\text {th }}$ International Conference on Insulated Power Cables (Jicable), Paper E8.5. 